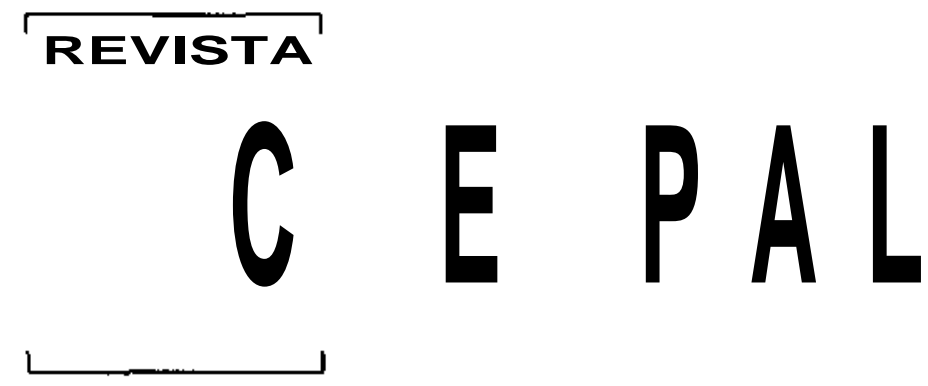

NUMERO 65

AGOSTO 1998

SANTIAGO DE CHILE

ÓSCAR ALTIMIR

Director

EUGENIO LAHERA

Secretario Técnico

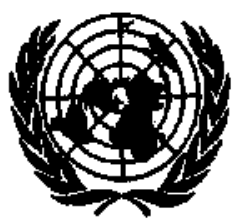

NACIONES UNIDAS 
Distribución del ingreso, pobreza y gasto social en América Latina José Antonio Ocampo

Gasto militar y el desarrollo en América Latina

Eugenio Lahera y Marcelo Ortúzar

Crecimiento, justicia distributiva y política social

Andrés Solimano

Equidad, inversión extranjera y competitividad internacional

Adolfo Figueroa

Tensiones en el ajuste estructural en América Latina: asignación vs. distribución

Daniel M. Schydlowsky

Competitividad y regulaciones laborales

Luis Beccaria y Pedro Galín

Familias latinoamericanas: convergencias y divergencias de modelos y políticas

Irma Amagada

Los acuerdos de libre comercio y el trabajo de las mujeres:

el caso de Chile

Alicia Frohmann y Pilar Romaguera

Evolución macroeconómica del Paraguay 1989-1997:

burbuja de consumo y crisis financiera

Stéphane Straub

Estrategias de las empresas mexicanas en sus procesos de internacionalización

Alejandra Salas-Porras

La regulación de la prestación privada de servicios de agua potable y alcantarillado

Terence R. Lee y Andrei S. Jouravlev

Promoción de la calidad para mejorar la competitividad $169 v^{\prime}$ Hessel Schuurman 


\section{Competitividad y regulaciones laborales}

Luis Beccaria

Pedro Galín
En este artículo se analizan ¡as relaciones entre la competitividad de una economía y las regulaciones laborales. Se argumenta que la teoría económica no es concluyente respecto del impacto de las regulaciones laborales en la competitividad, ya que diversas corrientes sostienen posiciones contradictorias en muchos aspectos. Por otra parte, las investigaciones empíricas han mostrado la escasa relevancia de la información aportada respecto a esas presuntas vinculaciones. De lo anterior se desprenden consecuencias de política: los países tienen ante sí diversas estrategias y mayores márgenes de maniobra de lo que suele sugerirse, ya que muchas políticas destinadas a mejorar la equidad no significan necesariamente restricciones a la competitividad. Un país puede, por tanto, elegir el nivel deseado de protección social. El mejoramiento de la competitividad se convierte, en parte al menos, en un tema de distribución del ingreso. La receta más convencional postula la reducción de la calidad de vida de los trabajadores por un período cuya extensión es difícil prever, con el fin de dotar a las empresas de ciertas condiciones iniciales que les permitan compensar las diferencias de productividad. Pero esta receta no es la única, ni es la más equitativa o eficaz, Para generar esas condiciones se pueden diseñar esquemas de redistribución del ingreso que hagan atractiva la inversión para competir internacionalmente, sin recurrir a nuevas erosiones de las remuneraciones de quienes fueron precisamente jos más afectados durante los últimos años de aplicación de los modelos sustitutívos. El análisis efectuado sugiere que son amplios los márgenes de libertad para optar entre diversas combinaciones de equidad y eficiencia, y que su selección depende de las relaciones de fuerza de los diversos actores sociales y de la estructura del Estado. 


\section{I}

\section{Introducción}

La competitividad y las regulaciones laborales, temas de este artículo, dicen relación con cuestiones de gran amplitud, considerable antigüedad y enormes repercusiones.

No se trata de las relaciones entre dos ciencias (como la economía y el derecho del trabajo), ${ }^{1}$ ni entre dos hechos, sino entre un fenómeno económico - la competitividad - y el derecho del trabajo, concebido aquí como derecho positivo y de alcance amplio (comprendiendo a la seguridad social). Desde luego, las regulaciones laborales implican no solamente costos: sus funciones principales incluyen la protección tanto de los trabajadores como de la producción, ya que organizan a esta última y establecen la "legalidad industrial". Si bien el tema se encaró aquí con un enfoque interdisciplinario, cabe señalar que en tanto la literatura económica aporta un enorme bagaje para abordarlo, el derecho del trabajo cuenta solamente con contribuciones más bien aisladas.

En lo que se refiere a la antigüedad de la materia, vale recordar que en el debate sobre las leyes que limitaron la jornada de trabajo se argüyó con frecuencia que serían ruinosas para las industrias del país que las adoptara por la competencia internacional de aquellos que no aplicasen esa normativa. En el preámbulo de la Constitución de la Organización Internacional del Trabajo se afirma "que si cualquier nación no adoptare un régimen de trabajo realmente humano, esta omisión constituiría un obstáculo a los esfuerzos de otras naciones que deseen mejorar la suerte de los trabajadores en sus propios países". La propuesta británica inicial para la constitución de la OIT señalaba que uno de los principales objetivos de la nueva organización era eliminar la competencia basada en condiciones de trabajo inhumanas. ${ }^{2}$ También los debates y argumentos actuales encuentran los ecos de polémicas casi remo-

Una versión preliminar de este trabajo fue presentada a las IX Jomadas Rioplatenses de Derecho del Trabajo y de la Seguridad Social (Punta del Este, II y 12 de mayo de 1996).

1 Parecería que el derecho del trabajo no ha sido ajeno a una ofensiva "como no se ha había visto desde la década de 1890: una tendencia deliberada a imponer el monopolio del método económico a todos los estudios de la sociedad", que Adam Prezworsky advertía ya hace una década (Prezworsky, 1987, p. 97).

2 Cilado por Caire, 1994. tas: en una reciente publicación se reprodujo un artículo publicado originalmente en 1927, incluido para demostrar que en esos tiempos los problemas económicos no eran totalmente diferentes de lo que son hoy día. ${ }^{3}$

Las repercusiones del tema no son exclusivamente académicas. Por el contrario, en torno a él se despliegan los intereses y las posiciones de diversas fuerzas sociales y políticas y de los Estados, enfrentados en interpretaciones acerca de los valores de la justicia social, el proteccionismo, la competencia internacional e incluso la preservación de la civilización. El debate internacional más intenso se presenta actualmente en torno a la "cláusula social" o las llamadas dimensiones sociales de la liberalización del comercio internacional, una cuestión que merece un estudio específico y no será abordada aquí.

Estas tres características: amplitud, antigüedad e importancia del tema, forzarán a recortar los objetivos del trabajo: se mostrará aquí lo que se sabe actualmente sobre la materia sin pretender efectuar contribuciones originales ni aportes empíricos sustantivos.

El plan de exposición será el siguiente: en la sección II siguiente se presentarán las aproximaciones principales de la teoría económica, frecuentemente estilizadas tanto por razones de espacio como institucionales: se omitirán exposiciones cconométricas, arduas y tediosas para el contexto en el que se presentan. En la sección III se plantean los argumentos más recientes esgrimidos en el debate específico sobre las regulaciones laborales. Los efectos de tales regulaciones que señalan los estudios empíricos se examinan en la sección IV, incluyendo los aspectos más salientes de la forma en que el tema está siendo abordado en la Argentina. La sección V, por último, entrega algunas reflexiones finales.

En lo referente a las fuentes, los aportes son preponderantemente de literatura económica, ya que son pocos los estudios jurídicos del tema. ${ }^{4}$

\footnotetext{
3 El trabajo de 1927 es el de Herbert Feis, reproducido en Sengerberger y Campbell, eds. (1994, pp. 29-55).

${ }^{4}$ En la producción jurídica argentina solamente hemos encontrado dos artículos: el de Armando Caro Figueroa, 1922, p. 383 y ss., y el de Geraldo von Potobsky, 1995.
} 


\section{II}

\section{Teoría económica y la intervención del Estado}

Esta sección plantea de manera breve los argumentos económicos básicos acerca de la intervención estatal con el fin de brindar un marco de referencia al tratamiento más específico de los impactos de las regulaciones laborales sobre la competitividad.

Respecto a la intervención del Estado, el pensamiento neoclásico afirma que el mercado - operando sin interferencias- asegura el logro del máximo bienestar de la población. Ofrece, sin embargo, dos justificativos para la intervención estatal; por un lado, la presencia de fallas de mercado: existencia de monopolio u oligopolio o monopsonio u oligopsonio, externalidades, falta de información, o el hecho de que se trate de bienes públicos o meritorios. Por el otro, la decisión de alterar la distribución del ingreso cuando el resultado de la libre operación de los mercados se considera inadecuado. Estrictamente, se trata de aquellas intervenciones que intentan mover a la economía de un óptimo - desde el punto de vista de la eficiencia- a otro.

Los economistas neoclásicos señalan, sin embargo, que la acción del Estado no siempre mejora el bienestar agregado, aun cuando existan fallas de mercado o la distribución resulte manifiestamente inequitativa. Así por ejemplo, se argumenta usualmente que muchas intervenciones basadas en el objetivo de redistribución no mantienen al sistema en la frontera de las posibilidades, sino que lo llevan a puntos por debajo de ella, incurriendo en una reducción de la eficiencia global. Aquellas acciones fundadas en la existencia de fallas de mercado pueden ser también cuestionadas debido a que existirían "fallas del gobierno", especialmente porque las intervenciones públicas se diseñan teniendo principalmente en cuenta los objetivos de políticos o burócratas, lo cual lleva a pérdidas de eficiencia mayores que las que derivan de la presencia de externalidades, bienes públicos o monopolios.-"'

En un contexto dinámico, los neoclásicos afirman que la interferencia del Estado lleva a una asignación inadecuada que afecta la capacidad de crecimiento de la economía al reducir la rentabilidad de las inversio-

\footnotetext{
5 Según Self (1993), los autores neoclásicos hacen hincapié en las muchas oportunidades que tienen los burócratas para pervertir los objetivos políticos en su propio beneficio.
}

nes, desestimular el ahorro o dar señales que llevan a disminuir la inversión en aquellas actividades con mayor capacidad de crecimiento. Asimismo, en una economía abierta, la intervención estatal también alteraría los incentivos, con lo cual, nuevamente, se derivarían demasiadas inversiones hacia las ramas para las que se cuenta con menores ventajas, generando así un uso ineficiente de los recursos, disminuyendo la competitividad global de la economía y reduciendo su capacidad de crecimiento.

Los planteos críticos a estos postulados básicos del pensamiento neoclásico realizados por autores neoclásicos heterodoxos o no neoclásicos se ubican en dos niveles de análisis. En primer lugar, existen diferencias conceptuales acerca del impacto de las regulaciones y las instituciones sobre la eficiencia económica. En segundo lugar, hay controversias sobre cuestiones empíricas referentes a la existencia misma de ciertas fallas de mercado o a la importancia cuantitativa que sobre la eficiencia pueden llegar a tener diversas intervenciones.

En lo que hace a la discusión conceptual, se pone de relieve que ciertas instituciones tienen un impacto positivo sobre la eficiencia; se ha planteado también que los precios no sólo constituyen una señal para la asignación de recursos sino que influyen en la calidad del producto. En estas circunstancias, el libre juego del mercado no siempre logra automáticamente un óptimo (Stiglitz, 1987). También puede existir la posibilidad de conflicto entre el corto plazo (ineficiencia asignativa) y el largo plazo (incremento de la productividad). Las políticas comerciales proteccionistas fueron justificadas precisamente por el argumento de la industria incipiente o, más genéricamente, por la necesidad de "crear" ventajas competitivas.

Otra idea contrapuesta a la visión ortodoxa es que los factores determinantes económicos dejan un margen más o menos amplio para que los actores involucrados - compradores o vendedores, trabajadores y empresarios- negocien los niveles de precio y cantidad sin que ello perjudique la eficiencia. Esto significa que en lodos los casos los compradores y vendedores tienen cierto poder de negociación, con lo cual resultan necesarias diversas instituciones que regulen los intercambios y equilibren las naturales diferencias de capacidad competitiva entre los actores. 
Por el lado de la discusión sobre la existencia misma de fallas de mercado, el pensamiento keynesiano señala que muchas de ellas — por ejemplo, la capacidad de ejercer algún grado de control del mercadoson muy relevantes e impiden el ajuste hacia el ópti- mo. En cuanto a la evaluación de las distorsiones que causaría la regulación, diversos trabajos han mostrado que algunos de sus costos en términos de ineficiencia no son significativos frente a su claro impacto positivo en la distribución del ingreso.

\section{III}

\section{Las regulaciones laborales}

En la sección anterior se ha efectuado una breve reseña de las posiciones neoclásicas —así como de algunas corrientes críticas de esa visión ortodoxa- respecto de la intervención del Estado en general. Esta sección se centrará específicamente en el desarrollo de las diversas posturas analíticas en lo concerniente a las regulaciones laborales.

Los arquetipos de la visión ortodoxa en la materia serían, en lo que hace a la oferta, que: "todo programa que protege a la población de las consecuencias del desempleo provee un incentivo para no trabajar y es una traba para la economía" y, en lo que se refiere a la demanda, que "la asistencia social cambia las decisiones de empico en forma socialmente ineficiente" (Blank y Freeman, 1994).

Desde la perspectiva ortodoxa, los programas de asistencia a los desempleados o las prestaciones de la seguridad social pueden conducir a los trabajadores a elegir el ocio antes que el trabajo hasta que tales beneficios terminen; esto incrementa el bienestar de los beneficiarios, pero reduce el producto social global y prolonga el desempleo. Los programas que establecen beneficios sociales obligatorios pueden constreñir la elección de los trabajadores, determinando resultados subóptimos e ineficientes, así como costos laborales más elevados. Los beneficios por enfermedad generosos pueden producir una epidemia de dolores de cabeza, de espalda y males similares hasta que los días con cobertura sean completamente utilizados.

En términos de la demanda de trabajo, se estima que las leyes que limitan el reclutamiento o el despido de trabajadores, por ejemplo, pueden distorsionar las señales del mercado laboral y reducir la rentabilidad en razón del "exceso de trabajadores" (por empleo redundante) o conducir a los empresarios a contratar menos trabajadores en razón de las expectativas de mayores costos futuros. Las leyes que establecen impuestos a los empleadores para financiar la jubilación o las prestaciones por desempleo pueden inducir a las empresas a ocultar remuneraciones o hacer antieconómico contratar trabajadores de bajos ingresos, dependiendo de la incidencia de tales impuestos. Los salarios mínimos determinados legalmente pueden reducir el empleo en actividades u ocupaciones de salarios bajos.

Desde esta perspectiva se subrayan los efectos distorsionantes que sobre el ahorro y la inversión tienen los impuestos necesarios para financiar tales programas. Estos desvían capital de alternativas más fructíferas de inversión y, por tanto, si estas alternativas tuvieran mayor potencial de creación de empleos, el gasto monetario en sostener familias de bajos ingresos podría reducir permanentemente las oportunidades de empleo, dejando tanto a esas familias como al resto de la sociedad en peores condiciones en el largo plazo. Muchos de los programas expanden el empleo público, lo que refuerza las rigideces del mercado de trabajo.

Por último, la ortodoxia plantea un impacto negativo adicional en una economía abierta si los programas de protección social tienen alguno de los efectos negativos mencionados: al incrementar el costo laboral las normas protectoras pueden reducir la competitividad internacional. Sobre esto se volverá más adelante.

La visión opuesta a la neoclásica ortodoxa, que se nutre - como ya se ha señalado- de diversas corrientes teóricas, articula una serie de reservas respecto de posiciones como las recién reseñadas.

Un primer argumento resalta el papel de los programas en el incremento del capital humano y la productividad en el mercado de trabajo. Desde esta perspectiva, las normas laborales pueden crear incentivos de largo plazo para la inversión en formación (Blank y Freeman, 1994; Standing, 1992; Boyer, 1994). En la medida en que existan restricciones al despido de trabajadores, se induce a los empleadores a invertir más 
en la capacitación de la fuerza de trabajo, en tanto estas leyes crean relaciones de largo plazo que hacen rentable la inversión en adiestramiento específico.

En otros sentidos, las vinculaciones de largo plazo pueden inducir una mayor productividad de los trabajadores a través de, por ejemplo, la identificación con los objetivos de la empresa y su lealtad a ella.

De alguna manera, la teoría de los salarios de eficiencia también argumenta a favor de los efectos benéficos de un tratamiento equitativo de los trabajadores. De acuerdo con esta visión, las remuneraciones constituyen no sólo un mecanismo de asignación sino también un medio para incentivar la eficiencia de la fuerza de trabajo. Consecuentemente, impedir que la tasa de salarios baje demasiado tendría un efecto positivo sobre la productividad. En particular, algunas de sus versiones ven la actitud de los asalariados en forma diferente a la neoclásica: el trabajador no intenta reducir el esfuerzo que le demanda desarrollar su tarea - visión implícita en la idea de que ese esfuerzo merma la utilidad del individuo - sino que realiza siempre un esfuerzo dado, el cual puede ser influenciado por la remuneración que reciben: "los trabajadores que se consideran tratados equitativamente seguramente trabajarán más duro" (Akerlof y Yellen, 1988, pp. 45).

El efecto de salarios mínimos, criticados —como se mencionó- por reducir la demanda de trabajadores con menor calificación, tendría un efecto benéfico sobre la eficiencia al incrementar la oferta de esos grupos.

Por otra parte, los argumentos ortodoxos acerca de los efectos distorsionantes de las regulaciones suelen tomar como marco de referencia un mundo de competencia perfecta, sin analizar el contexto económico global, en el que existen fallas de mercado y otras regulaciones. Un ejemplo lo brinda la legislación británica sobre igualdad de remuneraciones de principios de los años setenta: contrariando la prescripción neoclásica, no hay pruebas de que el incremento relativo de los salarios de las mujeres que provocó esta norma se haya traducido en una reducción del empleo femenino, lo cual pudo deberse a que no se verificaba plenamente el paradigma de la competencia perfecta y los empleadores ejercían algún grado de control del mercado. En ese contexto, la norma permitió incrementar la eficiencia global (Gregg, Machin y Manning, 1994, pp. 109).

Desde una perspectiva más empírica, algunos autores heterodoxos señalan que si bien las normas protectoras tienen efectos distorsionantes sobre los mercados de trabajo, no se miden los beneficios que ellas generan, los que por lo demás resultan difíciles de cuantificar (Blank y Freeman, 1994, pp. 31 y 32; Sengenberger y Campbell (eds.) 1994, pp. 13-14; Standing, 1992). Los beneficios económicos de las normas laborales - algunos de los cuales se mencionaron más arriba- son indirectos, ocultos, intangibles, dilatados en el tiempo (Boyer, 1994, p. 26) y no localizados. Así, se ha presentado el ejemplo de los costos impuestos por las normas sobre higiene y seguridad, los que son por lo general fáciles de estimar. También lo son algunos de los beneficios que generan (ahorro de erogaciones, por ejemplo, en el cuidado de los trabajadores accidentados y en su reemplazo) y que pueden superar dichos costos. Más difícil es estimar la pérdida de productividad y de poder de compra, así como lo perdido en los costosísimos accidentes industriales recientes (Sengenberger y Campbell, eds., 1994), para no mencionar valores humanos no mensurables económicamente como la vida y la salud. Muchos de estos fenómenos generan además externalidades negativas que en general son difíciles de computar. Para hacer un completo análisis costo-beneficio de cada programa social es preciso contraponer el incremento en el bienestar económico de los ciudadanos con sus costos directos e indirectos. La simple demostración de los efectos distorsivos o costos de inefíciencia no es suficiente (Blank y Freeman, 1994, p. 32).

Esto último apunta también a que los argumentos ortodoxos no suelen tener en cuenta el efecto de las normas e instituciones protectoras sobre la distribución del ingreso. Instituciones como ios sindicatos no sólo cumplen un papel importante desde el punto de vista de la eficiencia, al reducir los costos de transacción, sino que tienden a contrarrestar la natural diferencia de capacidad negociadora que existe entre trabajadores y empleadores. Incluso, en tanto los empleadores reconozcan cierto poder del mercado, como se señaló en un párrafo anterior, los sindicatos aumentan y no disminuyen la eficiencia.

Incluso en términos de la teoría económica ortodoxa, sostienen los heterodoxos, la afirmación de que las normas laborales o los programas sociales reducen el crecimiento económico es débil. Los análisis indican una pérdida de eficiencia en términos estáticos, pero no una reducción de las tasas de crecimiento. La teoría del crecimiento no brinda predicciones claras respecto a cómo las distorsiones estáticas alteran el ritmo de desarrollo económico. Más aún, conforme a diversos argumentos arriba mencionados, las ineficiencias de corto plazo pueden ser compensadas por la eficiencia dinámica. 
Conviene concluir esta sección con comentarios específicos sobre el tema de la competitividad. La capacidad de competir no depende sólo de la eficiencia o productividad relativa, Un país puede inundar a otro con bienes que produce menos eficientemente si la diferencia en los precios de los insumos y factores que intervienen en la producción es mayor que la diferencia en la eficiencia con que esos insumos o factores son empicados. ${ }^{6}$ Desde una perspectiva agregada esto significa que un país puede ser "competitivo" a partir de salarios bajos. Por tanto, una productividad más baja no implica siempre una menor posibilidad de competir sino menor calidad de vida.

La ortodoxia sostiene que la baja relativa de la productividad puede ocasionar una disminución de la competitividad sólo temporal, en tanto los mecanismos de mercado provoquen una reducción de los salarios y/o los precios de otros factores (bajan las exportaciones y/o suben las importaciones, lo cual promueve una caída de la producción y el empleo) y tienda a recomponerse la competitividad. Como este mecanismo autorregulador estaría en parte impedido por la existencia de normas laborales, propone reducir tales normas: esto provocaría un menor costo salarial que al mejorar la competitividad haría atractivo un proceso de inversión; éste, con el tiempo, elevaría la productividad y —en condiciones de crecimiento del empleotambién los salarios.

La crítica heterodoxa a esta propuesta señala en primer lugar que el mejoramiento de la competitividad basado en la baja de salarios: i) lleva a no profundizar la búsqueda del incremento de la eficiencia, ii) promueve una especialización en bienes relativamente simples, que requieren mano de obra poco calificada y que suelen tener menor futuro, y iii) no constituye en sí una estrategia al no poder repetirse y al verse sus efectos superados por los incrementos de productividad de los otros países. En segundo lugar, recurre a los ya mencionados cuestionamientos a la idea ortodoxa de que la desregulación y los menores salarios favorecen el crecimiento de la productividad.

\section{IV}

\section{La información empírica}

Una vez presentados de manera esquemática los argumentos teóricos en torno a la incidencia de las normas laborales en la competitividad, es conveniente encarar la información empírica arrimada para sostener las respectivas posiciones. Por lo general, los datos se han elaborado teniendo en cuenta el comportamiento disímil de los países desarrollados, particularmente los de la Organización de Cooperación y Desarrollo Económicos (OCDE), por un lado, y los del Sur, por el otro.

Una afirmación general sintetiza las conclusiones de esta sección: al revisar los argumentos acerca de las relaciones entre los programas de protección social, la flexibilidad del mercado de trabajo y el bienestar económico agregado, se observa que es poca la información disponible sobre numerosas cuestiones (Blank y Freeman, 1994, p. 36).

\footnotetext{
${ }^{6}$ La mayor competitividad que exhibe una actividad determinada no se deriva exclusivamente de su mayor eficiencia y/o de ios menores precios que rigen en ella. También puede obedecer a los menores precios que paga por insumos (en cuya producción se alcanza una elevada eficiencia y/o se abonan bajos precios a los factores) o bienes de consumo (que lleva a que los salarios sean reducidos).
}

\section{Países industrializados}

Respecto al mundo desarrollado, se han establecido comparaciones entre, por un lado, las economías europea - en especial las de la Unión Europea - con reglamentaciones y protección social nutridas, y los Estados Unidos, con prestaciones de seguridad social y regulaciones laborales reconocidamente más laxas. Con frecuencia también ha entrado en el análisis el Japón, que cuenta con normas laborales y sistemas de protección social más rígidos que los estadounidenses, aunque el nivel de negociación colectiva es descentralizado.

En principio, la ortodoxia ha considerado a los Estados Unidos como país paradigmático de la competitividad por sus escasas regulaciones, en tanto que la CEE encarnaría el opuesto.

El estudio probablemente más sistemático proveniente de canteras ortodoxas es aquel que analiza el comportamiento de los países de la OCDE signatarios de acuerdos comerciales regionales: esto es de la Comunidad Económica Europea (CEE), de la Asociación Europea de Libre Intercambio (AELI), integrada por los 
países escandinavos, Suiza y Austria, y del Tratado de Libre Comercio de Norteamérica (NAFTA O TLCN). ${ }^{7}$ En dicho estudio se analizan las "reglamentaciones y disposiciones relacionadas con el tiempo de trabajo, los contratos, los salarios mínimos y los derechos de representación de los trabajadores", concluyendo que "no existe una relación única y directa entre las normas laborales y los resultados comerciales (...) Sin embargo, es demasiado pronto para extraer esta conclusión: es necesario realizar más investigaciones empíricas" (OCDE, 1995). Un estudio más reciente de la misma organización (OCDE, 1996) indica las dificultades con que se tropieza para analizar empíricamente las vinculaciones entre las normas laborales fundamentales y los flujos comerciales; la principal limitación es que la información relativa a las normas fundamentales es escasa e incompleta, salvo en lo que se refiere a la libertad sindical y la negociación colectiva. Las conclusiones de este trabajo se refieren principalmente a dichas normas fundamentales. La información empírica confirma los resultados analíticos según los cuales las normas laborales fundamentales apenas influyen en los desempeños comerciales. La idea de una nivelación hacia abajo que haría que los países con normas mediocres ganen porciones de mercado de exportación en detrimento de aquellos con buenas normas no está confirmada por la observación empírica. De conformidad con estas conclusiones, se afirma que los países en desarrollo no tendrían justificación empírica para pensar que una mejoría de las normas laborales fundamentales perjudicaría su desempeño económico o su competitividad en ios mercados mundiales.

El escepticismo respecto de las correlaciones entre normas protectoras y productividad es compartido por otros organismos internacionales. Así, un documento oficial de la OIT afirma: "procede contrastar... las ventajas teóricas de una competencia declarada y descentralizada en los mercados de trabajo con la constatación de que las tres economías más prósperas del mundo, Estados Unidos, Japón y Alemania, tienen sistemas de fijación de los salarios, índices de densidad sindical, niveles de negociación y modalidades de coordinación de toda la economía muy diferentes. La conclusión más elemental es que tales disparidades no cuentan en lo que se refiere al empleo global y otras variables macroeconómicas" (OIT, 1995). En la Comi-

\footnotetext{
7 En verdad, México no se analiza por insuficiencia de información.
}

sión Europea se han elaborado documentos de distinto tenor. En uno de ellos se señala que "Existe unanimidad en lo relativo al mal funcionamiento de los mercados de trabajo. El origen de la rigidez es la falta de flexibilidad del mercado de trabajo, en especial desde el punto de vista de la organización del tiempo de trabajo, de los salarios y de la movilidad..." (Comisión Europea, 1994a), En otro documento oficial la "unanimidad" sobre el tema aparece cuestionada: "hay quienes arguyen que una normativa laboral excesivamente restrictiva trae consigo costes que reducen el carácter competitivo de empresas en un país o región respecto a otras. Por otra, muchos creen que la productividad y que las altas normas laborales siempre han sido parte integrante de la fórmula competitiva. La tensión entre estos dos puntos de vista ha sido muy evidente... Hay que decir que no hay ningún consenso claro en este punto.." (Comisión Europea, 1994b). Un estudio publicado por la Conferencia de las Naciones Unidas sobre Comercio y Desarrollo (UNCTAD) afirma que no existe información empírica que permita refutar o apoyar la tesis de que los derechos laborales laxos conducen a una ventaja competitiva comercial, por lo que es preciso profundizar los estudios sobre cada una de las normas en consideración (De Castro, 1995, pp. 9 y 10),

En el ámbito académico tampoco se encuentran estudios que aporten datos concluyentes al respecto. En el iv Congreso Regional Europeo de la Asociación Internacional de Relaciones Industriales se examinaron las ventajas competitivas de las relaciones industriales europeas, estadounidenses y japonesas. El relator oficial indicó que "los documentos preparados...se caracterizan por las actitudes cautelosas y los juicios reservados al evaluar las ventajas y los desarrollos potenciales de los bloques europeo, japonés y americano. De acuerdo con esos documentos, ninguna de las propiedades que se encuentran tras los tres grandes bloques mundiales tiene una superioridad general sobre sus competidores, teniendo cada uno de ellos sus méritos y desventajas relativas" (Jacobi, 1994, p. 18). En otro estudio se ponen de relieve las complejidades del tema y las "simplificaciones" en que incurrirían las diversas interpretaciones, aunque en algunos casos ciertas reglamentaciones laborales, como las normas italianas extremadamente rígidas sobre reclutamiento o la falta de normas que protejan la seguridad en el empleo en Estados Unidos, pueden ser asociadas con una pérdida en la eficiencia económica global (Buechtemann, 1993, p. 62), Otros estudios, por su parte, indican que los Estados Unidos, el país industria- 
lizado con normas laborales más flexibles y menor protección social y que durante decenios tuvo la productividad más alta, perderá ese liderazgo hacia el año 2000, superado por Francia y Bélgica, ya que su ritmo de incremento de la productividad es inferior al de los países de la OCDE, salvo Australia y los Países Bajos (Freeman, 1994, p. 9). Tampoco son categóricas las conclusiones de un estudio comparativo de cinco países industrializados (Estados Unidos, Francia, Gran Bretaña, Alemania y Suecia) sobre la competitividad y el estado de bienestar; ",.,1a falta de disciplina económica hace que el Estado de Bienestar entre en conflicto con la competitividad de los precios... pero por el contrario, la productividad y la calidad se benefician del Estado de Bienestar..." (Pfaller, Gough y Therborn, comps., 1993, p. 368 y ss.). En otra investigación más reciente se afirma que la teoría y la información empírica proveen pocas razones para esperar que las diferencias en la normativa laboral contribuyan significativamente a diferencias en los costos de producción $\mathrm{y}$, con ello, a las pautas de inversión y comercio. ${ }^{8}$

Dada la extensa gama de trabajos examinados, cabe sugerir que no existe información empírica que sustente las supuestas vinculaciones entre normas laborales y competitividad, ni siquiera en lo que hace al sentido en el que operan esas interrelaciones.

\section{Países en desarrollo}

Se ha argumentado intensamente que la liberalización del mercado de trabajo es un ingrediente esencial de los programas de ajuste estructural en los países en desarrollo, necesarios para dinamizar la economía e integrarla exitosamente al mercado mundial, mejorando su competitividad. Esta tesis ortodoxa ha entendido que "la excesiva reglamentación del mercado de trabajo en América Latina ha exacerbado el dualismo estructural entre un sector urbano muy protegido y un sector estructurado que lo está apenas y que ese exceso de reglamentación ha impedido que el mercado de trabajo se adaptara a la evolución de las fuerzas del mercado mientras que en los países asiáticos de industrialización reciente el mercado de trabajo está muy poco reglamentado, a lo cual se debe el éxito de las estrategias de desarrollo de las exportaciones". A ese éxito habría contribuido la represión de los sindicatos,

\footnotetext{
8 K. Anderson, The enlwining of trade policy with environmental and labour standards, documento presentado a la Conferencia del Banco Mundial "Uruguay Round and the Developing Economies" (26 a 27 de enero de 1995), citado por De Castro, 1995, p. 10).
}

sobre todo en su función de fijación de los salarios (OIT, 1995, p. 93).

Sin embargo los heterodoxos han señalado que en vista de esas intervenciones represivas es un "poco ingenuo pretender que se evitaran las distorsiones de precios en los casos exitosos de crecimiento industrial" (Standing, 1992, p, 26). De otro lado, se ha dicho que la represión a los sindicatos no fue generalizada (Hong Kong respetó las tradiciones liberales británicas y su comportamiento económico fue tan exitoso como el de los otros "tigres")- En Singapur y la provincia china de Taiwán, los modelos de intervención en los sindicatos fueron distintos: se los ha mantenido controlados a través de un partido único. El movimiento sindical de Singapur, gracias a su participación en el Consejo Salarial Nacional, ha podido ejercer cierta influencia en las políticas económicas y sociales, dentro de límites reconocidamente estrechos. De todas formas, con la intervención en los años setenta, que tendió a restringir las alzas salariales para que se mantuvieran por debajo del incremento del PIB, también se establecieron políticas de corrección salarial para enfrentar la escasez de mano de obra y estimular a las empresas a emprender actividades de tecnología más compleja.

Ahora bien, se ha planteado si estas pautas de control del mercado laboral en el sudeste asiático eran necesarias para el éxito competitivo. Como señala el mismo Banco Mundial, "En general, los trabajadores están más dispuestos a aceptar la flexibilidad salarial con una tendencia rápida al alza, porque un ajuste a la baja implica un ritmo más lento de aumento y no un descenso absoluto de los ingresos laborales reales". 9 Esto es, la elevación constante de los salarios reales observada en los "tigres" asiáticos habría facilitado la aceptación generalizada de la flexibilización salarial, aunque el ritmo de incremento haya sido menor al de la productividad. Por otra parte, el grado de intervención del Estado en la economía fue muy intenso, con políticas industriales muy activas y de carácter coercitivo, que podrían haber influido mucho más decisivamente en el éxito económico que la desregulación del mercado de trabajo, o mejor dicho, su regulación represiva o paternalista, como dice Standing (1992, p. 38). Se ha afirmado que uno de los más importantes rasgos del desarrollo económico sudcoreano es

\footnotetext{
* G. Fields, "Changing labor makel conditions and economic development in Hong Kong, the Republic of Korea, Singapore and Taiwán", en World Bank Economic Review, Vol 8. ํㅜ 3, citado en OIT, 1995, p. 95.
} 
probablemente el fuerte papel del Estado, sobre todo en su capacidad para influenciar y disciplinar a fas grandes empresas que dominan la economía coreana (Park, 1994a, p. 209). "Lo que permitió a los países del este asiático disciplinar a sus empresas fue la relativa debilidad tanto de los grupos industriales como agrarios en los comienzos del desarrollo industrial de la posguerra". ${ }^{10}$

Por último, la creciente incorporación de instituciones protectoras —el salario mínimo en la República de Corea y la provincia china de Taiwán, y el seguro de desempleo en la primera-, así como el grado creciente de independencia del movimiento sindical, no parecen haber alterado el ritmo de crecimiento de esos países. "Esa evolución normativa en Asia pone, pues, de manifiesto que para el éxito en los mercados de exportación no es indispensable ni un mercado de trabajo totalmente liberalizado ni la represión de las reivindicaciones de los trabajadores" (OIT, 1995).

Otras visiones ponen en tela de juicio incluso que la intervención estatal en la República de Corea haya sido exclusivamente represiva, y consideran que deben tomarse en cuenta los importantes ingredientes paternalistas de las políticas laborales en los últimos treinta años. Con miras a ganar competitividad en los mercados internacionales, el trabajo fue extensamente regulado por el gobierno. Esto no significó necesariamente que la intervención en el mercado de trabajo se orientara exclusivamente al beneficio empresarial. Con el fin de prevenir la explotación de los trabajadores ante la ausencia de derechos laborales (colectivos), el gobierno estableció una serie de disposiciones protectoras e incluso alentó a los empleadores a mejorar las condiciones de trabajo hasta un nivel compatible con el grado de desarrollo económico (Park, 1994b), Un estudio del Banco Mundial ha hecho notar, incluso, que el gobierno sudcoreano intervino para asegurar que los salarios no se incrementaran por encima de la productividad y a la vez que los trabajadores participaran en los frutos del crecimiento (Mazumdar, 1994). Aunque se restringió la autonomía sindical, las revisiones de la ley laboral a comienzos de los años sesenta reforzaron la protección individual. A pesar de la severa opresión de los derechos colectivos, las revisiones de la ley del trabajo de 1970 incluyeron algunas medidas para acrecentar el bienestar de los trabajadores y en 1981 hubo una serie de disposiciones para mejorar las re-

10 A. Amsden, "A theory of government intervention in late industrialization", citado por Hikino y Amsden, 1995, p. 6. gulaciones de salud y seguridad. Un examen de la historia de la legislación laboral revela que el Estado continuó incrementando la protección de las relaciones individuales de trabajo, en tanto que al mismo tiempo reducía las disposiciones legales protectoras de las relaciones colectivas. Los salarios reales crecieron desde 1965 a una tasa anual acumulativa del 7.5\%, lo que superó el desempeño de todos los países industrializados, incluido el Japón, en los períodos de rápido crecimiento. La distribución del ingreso también mejoró sustancialmente, tornándose más igualitaria, y la participación de los ingresos de los asalariados en el ingreso nacional pasó del $31.8 \%$ en 1965 al 59.7\% en 1990. Si bien la jornada de trabajo sudcoreana es la más extensa y la tasa de incidencia de accidentes la mayor entre todos los países cuyos datos son entregados al Anuario de Estadísticas del Trabajo de la OIT, la extensión de la jornada ha decrecido significativamente desde 1980; en cuanto a los accidentes, éstos estarían siendo registrados en forma más completa que en otros países (Park, 1994b).

En términos más generales, se ha encontrado una fuerte correlación, en una submuestra de países de industrialización tardía, entre igualdad en la distribución del ingreso y el crecimiento de la productividad (Hikino y Amsden, 1995, p. 17).

La argumentación ortodoxa es bien conocida en América Latina: las barreras arancelarias y el elevado involucramiento del Estado, al alterar las rentabilidades relativas de los sectores, habrían generado economías con escasa capacidad de crecimiento y/o fuertes inestabilidades macroeconómicas. Las normas laborales, en particular, habrían quitado flexibilidad al mercado de trabajo, aumentado los costos no salariales y elevado desmedidamente, en algunos casos, la capacidad de presión de los gremios. Todo ello explicaría un costo salarial que no concordaba con la productividad alcanzada, lo que perjudicaba la competitividad de estas economías, así como su capacidad de generar ocupaciones productivas. Esto se habría traducido en un alto grado de subutilización de la mano de obra que se manifestaba en la elevada proporción de trabajadores informales; la segmentación resultante explicaba en gran medida la desigual distribución personal del ingreso, y la escasa dinámica productiva y de generación de empleo llevaba también a que, en el largo plazo, no pudiese crecer la participación de los salarios en el ingreso nacional.

Las investigaciones comparativas realizadas en el contexto latinoamericano comparten, sin embargo, el escepticismo respecto de la contundencia de la infor- 
mación disponible. Así, en un estudio del Banco Mundial se ha afirmado que si se contrasta, por ejemplo, el ajuste relativamente exitoso de Costa Rica y el problemático de Bolivia, queda claro que el desmantelamiento de las instituciones laborales no es ni necesario (Costa Rica) ni suficiente (Bolivia) para que un ajuste tenga resultados satisfactorios. Se agrega que en futuros estudios sería fructífero analizar el papel de las instituciones laborales, los sindicatos y la política económica, antes de lanzarse a una defensa global del desmantelamienlo de esas instituciones (Horton, Kanbur y Mazumdar, 1994, pp. 45 y 57),

Desde una óptica distinta se afirma, al analizar los regímenes de protección de México, Colombia, Perú, Argentina, Chile, Brasil y Uruguay, que "no es evidente que la legislación laboral tuviera efectos precisos sobre la productividad industrial global.,, el presente estudio confirma que el principio que inspira las actuales propuestas políticas en algunos países latinoamericanos - a saber, que basta con flexibilizar los despidos y los contratos de trabajo para mejorar los resultados económicos - se basa en una concepción muy equivocada" (Marshall, 1994, pp.76-77).

Un estudio aún más reciente intenta dilucidar si la reducción de los costos laborales es necesaria para mejorar la competitividad. El análisis se concentra en la evolución del sector manufacturero en el período 1990-1995 en Argentina, Brasil, Chile, México y Perú. Se concluye que aunque los costos laborales crecieron en cuatro de los cinco países estudiados, la productividad creció aún más. Esto demuestra que los costos laborales no son un impedimento para que la competitividad se eleve, ya que su evolución se encuentra determinada más por la productividad del trabajo que por los costos laborales. El estudio concluye que el incremento de los costos laborales medidos a precios internacionales — que pudo afectar la competitividadfue determinado principalmente por las políticas macroeconómicas que han establecido tipos de cambio sobrevaluados respecto del dólar y, por lo tanto, alteraciones de los precios relativos en detrimento de los bienes primarios e industriales. Esto hizo que el aumento de dichos costos laborales para los empleadores en el sector de bienes transables no se tradujera necesariamente en mejoras proporcionales del salario real de los trabajadores. El salario industrial creció en el período analizado, pero al final del mismo era inferior al de 1980 en Argentina (-21\%), México (-38\%) y Perú $(-57 \%)$, en tanto que era un $10 \%$ más alto en Brasil y un 29\% más alto en Chile (Tokman y Martínez, 1995). Esto es, no se cuenta con datos contunden- tes respecto de la asociación entre derecho del trabajo (expresado como costos laborales totales) y competitividad, ya que son otras las variables decisivas.

En síntesis, la información disponible en los estudios de los países del Sur tampoco es contundente respecto de las relaciones entre instituciones laborales y competitividad: tanto investigaciones provenientes del Banco Mundial como de la OIT cuestionan las visiones simplistas que asocian en forma directa la desregulación o la ausencia de normas con las ventajas competitivas. En todo caso, se precisan mayores investigaciones que puedan desbrozar las complejas intcrrelaciones entre la multiplicidad de variables que determinan la competitividad, incluso en el corto plazo, pero más aún en el largo, como sugieren los estudios sobre la República de Corea.

\section{Argentina}

La propuesta de abandonar el modelo de acumulación basado en la sustitución de importaciones y adoptar un esquema de mayor exposición a la competencia internacional se incluyó originalmente en la propuesta económica enunciada pocos días después del golpe de 1976. Sin embargo, sólo a partir de 1991 se puso en marcha un programa amplio que, profundizando algunas tendencias ya individualizadas desde años atrás, modificó sustancialmente las reglas del juego en lo que hace a la relación comercial y financiera con el resto del mundo y al papel del Estado en la economía.

En este contexto, las regulaciones laborales pasan a constituir un caso particular en el tema más amplio de la intervención estatal. La ortodoxia entiende que estas normas imponen elevados costos de producción y desestímulos a la inversión que habían afectado negativamente la competitividad del aparato productivo. La eliminación o adecuación de las regulaciones del mercado de trabajo debían, por tanto, formar parte del acomodamiento más general del marco normativo imprescindible para insertar adecuadamente a la Argentina en los mercados mundiales.

En particular, se señalaba que los costos no salariales eran altos en relación a otros países de la región, a las naciones del sudeste asiático y aún a algunas economías industrializadas. En un trabajo que compara la situación de los países del MERCOSUR, se señala que el costo laboral total supera al salario bruto en $39 \%$ en Argentina, 23\% en Chile y 52\% en Brasil (FIEL, 1992). Este mayor peso del salario indirecto se explicaría por los elevados impuestos al trabajo para financiar la previsión social y otras regulaciones como las 
asignaciones familiares, el fondo de desempleo o el costo del despido.

Las características de la negociación salarial y un sindicalismo fuerte habrían contribuido además a que los salarios fuesen elevados y no hayan respondido a la situación del mercado de trabajo o a los niveles de productividad. Por ejemplo, para casi todas las actividades examinadas en el citado estudio de FIEL, el salario bruto en Argentina era superior - entre 10 y $20 \%$, y más en algunos casos - al chileno y al brasileño.

Algunas normas, como las relacionadas con el despido, no sólo implicarían costos medios altos sino que, al ser contingentes, elevarían la incertidumbre y el riesgo de ¡a inversión. Las regulaciones sobre el despido restarían, además, flexibilidad al mercado de trabajo. Por lo tanto, la normativa no sólo habría contribuido a que el costo laboral fuese elevado al inicio de la convertibilidad, sino que pesaba negativamente sobre la capacidad de mejorar la eficiencia productiva. Se señalaba que "...la fuente principal de reducción de costos laborales unitarios debe buscarse en dos aspectos que se hallan íntimamente relacionados: la flexibilización de las condiciones laborales y el incremento de la productividad por persona ocupada" (Bour, 1995, pp. 196-197). Se estaría argumentando, entonces, que la mayor flexibilidad externa estaría asociada a una mayor productividad.

Los analistas ortodoxos, por lo tanto, reiteradamente han propuesto disminuir los costos y flexibilizar las normas para mejorar instantáneamente la competitividad y permitir que vaya mejorando la productividad. Ello reduciría, además, el precio relativo de la mano de obra y posibilitaría que, a igual crecimiento de la producción, el comportamiento del empleo fuese más dinámico.

El incremento de la desocupación - tendencia presente aun antes de la crisis internacional de fines de 1994 - fue expuesto por algunos como una de las evidencias del efecto de la rigidez y elevados costos derivados de las regulaciones laborales. Sin embargo, durante los primeros años de la convertibilidad se expandieron la producción, la productividad y las exportaciones. Aun cuando las importaciones aumentaron significativamente, no podría argüirse que las regulaciones hayan constituido un obstáculo importante para que las firmas se ajusten a las nuevas condiciones. Se ha afirmado, sin embargo, que el incremento de la productividad industrial se habría derivado, en buena medida, de una estrategia "defensiva" de las firmas (Kosacoff, 1993, pp. 50-55) y que no hubo una corriente importante y generalizada de inversiones, procurando beneficiarse de las nuevas reglas del juego. Este hecho podría explicarse por el elevado costo laboral inicial que hacía poco competitiva a las actividades de bienes transables y, por tanto, poco atractivo el invertir en ellas. Debe tenerse en cuenta, sin embargo, que el elevado costo salarial relativo argentino que se registraba al inicio de la convertibilidad - y que se mencionó más arriba- era consecuencia, en buena medida, del nivel del tipo de cambio real. En efecto, como se observa en el cuadro 1 que incluye datos para la industria, los costos laborales en dólares eran altos, pero el salario real continuaba en niveles históricamente deprimidos (aunque superiores a los de los años de la hiperinflación). Como ocurrió con otros países de la región (véase la sección IV, subsección 2) esta tendencia se fue ahondando durante los años 1991-1993, ya que mientras el salario crecía en relación a los precios de la canasta de consumo (muy por debajo de la productividad), ${ }^{11}$ los precios industriales permanecían prácticamente estancados —influidos por el tipo de cambio fijo-, los precios de los servicios se elevaban y también, en consecuencia, el salario nominal y la relación salarios/precios industriales y salarios/tipo de cambio. Este deterioro de los precios relativos de los bienes transables se desaceleró fuertemente a partir de 1994, por lo que en ese año los costos laborales tanto en pesos como en dólares aumentaron (cuadro 1) de manera más próxima al salario real y también a la productividad. ${ }^{12}$ Incluso, se argumenta que la devaluación del peso argentino respecto del real brasileño y la del dólar respecto de otras monedas fuertes, así como el mejoramiento de los precios de productos que exporta la Argentina fueron otros factores que a partir de 1994 potenciaron el efecto benéfico que sobre la competitividad venía teniendo el aumento de la productividad (Kacef y Machinea, 1995).

De cualquier manera, tampoco la mera comparación de salarios en términos de dólares representa un indicio adecuado del grado de competitividad ya que, como se señaló en las secciones II y III, también se debe tomar en cuenta las diferencias de productividad.

\footnotetext{
11 J. Schvarzer (1994) cuestiona las cifras oficiales de incremento de la producción industrial porque considera que el cambio en el componente de producción nacional, que se redujo en muchas ramas manufactureras, no es considerado en los cómputos de cuentas nacionales. Al recalcular el crecimiento del PBI industrial llega a un valor de $10 \%$ inferior al oficial para 1994. Desde esta perspectiva, el incremento de la productividad industrial que se incluye en el cuadro estaría sobreestimado.

12 Más aún, durante ese año comenzaron a regir rebajas de aportes patronales. Según Szretter (1995), esto provocó una reducción del costo salarial unitario (esto es, el que considera al efecto de la productividad).
} 
CUADRO 1

Argentina: Salario y productividad en la industria manufacturera

(Índices)

\begin{tabular}{lccr}
\hline & Salario real $^{\mathrm{a}}$ & Salario costo & Salario en dólares \\
\cline { 2 - 4 } $1980-1988$ & 137.8 & 90.8 & 76.9 \\
1991 & 100.0 & 100.0 & 100.0 \\
1993 & 105.7 & 137.1 & 135.6 \\
1994 & 109.5 & 144.6 & 146.3 \\
\hline
\end{tabular}

Fuentes: Monza (1993) y Szretter (1995).

a Salario nominal deflacionado por el índice de precios al consumidor.

b Salario nominal deflacionado por el índice nacional de precios mayoristas no agropecuarios.

En este sentido, el ya citado estudio de FIEL muestra que en algunos sectores las diferencias de los salarios en dólares entre la Argentina y otros países limítrofes eran más que compensadas por las brechas que se registraban en la relación producto/empleo.

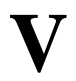

\section{Conclusiones}

A lo largo del trabajo se ha argumentado, primero, que la teoría económica no es concluyente respecto al impacto de las regulaciones laborales sobre la competitividad, ya que las posiciones de diversas corrientes son contradictorias en muchos aspectos. Las investigaciones empíricas han mostrado la escasa relevancia de la información aportada sobre esas presuntas vinculaciones. Esta conclusión puede obedecer, en parte, a la dificultad intrínseca de individualizar los efectos de las regulaciones.

De lo anterior se desprende, entre otras cosas, que los países tienen ante sí diversas estrategias y por tanto mayores márgenes de maniobra de los que suelen sugerirse desde las posiciones ortodoxas, ya que muchas políticas destinadas a mejorar la equidad no significan necesariamente restricciones a la competitividad. Un país puede, por tanto, elegir el nivel deseado de protección social. De hecho, las regulaciones laborales están lejos de ser similares, incluso en los estrechamente integrados países europeos (De Castro, 1995). El marco institucional de los diversos países es el resultado de numerosos factores históricos y culturales; en consecuencia, la gama de modificaciones factibles está fuertemente acotada por tales tradiciones.
En todo caso, ninguno de los estudios conocidos en el plano nacional ha aportado información empírica sustantiva sobre las repercusiones negativas de las regulaciones laborales en la competitividad (Feldman, 1995).

Así, el tema de cómo mejorar la competitividad trasciende el análisis meramente técnico y se convierte, en parte al menos, en un examen de la distribución del ingreso. La receta ortodoxa, que no parece tener suficiente sustento conceptual ni empírico, se basa en la necesaria reducción de la calidad de vida de los trabajadores por un período cuya extensión es difícil prever. Si bien un país que debe incrementar su relaciones comerciales y que lo hace con una base productiva atrasada debe dotar a las empresas de ciertas condiciones iniciales que les permitan compensar las diferencias de productividad, esta receta no parece la única, y de seguro no es la más equitativa ni necesariamente la más eficaz. Para generar esas condiciones parece posible diseñar esquemas de redistribución del ingreso que hagan atractiva la inversión para competir internacionalmente sin recurrir a nuevos deterioros de las remuneraciones de quienes fueron precisamente los más afectados durante los últimos años de políticas sustitutivas.

La cuestión de las vinculaciones entre las regulaciones laborales y la competitividad pudo ser vista como un conflicto entre la equidad y la eficiencia. 
Como hemos tratado de mostrar aquí, se trata en realidad de diversas concepciones económicas y jurídicas de equidad y de eficiencia. La perspectiva ortodoxa, al menos tal como se difunde corrientemente, sostiene que existe un solo y único camino para optimizar la eficiencia y que él implicaría sacrificios transitorios de equidad. La revisión efectuada sugiere que son amplios los márgenes de libertad para optar entre diversas combinaciones de equidad y eficiencia, y que su selección depende de las relaciones de fuerza de los diversos actores sociales y la estructura del Estado, antes que de fórmulas infalibles, exclusivas y certeras.

\section{Bibliografía}

Akerlof, G. y J. Yellen (1988): Fairness and unemployment, American Economic Review, vol. 78, № 2, Nashville, Tennessee, American Economic Association.

Blank, R. y R. Freeman (1994): Evaluating the connection between social protection and economic flexibility, R. Blank (ed.). Social Protection Versus Economic Reality: Is There a TradeOff!, Chicago, Illinois, Chicago University Press.

Bour, J. (1995): Los costos laborales en Argentina, MTSS, El libro blanco del empleo en la Argentina, Buenos Aires, Ministerio de Trabajo y Seguridad Social (MTSS).

Boyer, R. (1994): Do labour institutions matter for economic development? A "regulation"approach for the OBCD and Latin America, with an extensión to Asia. G. Rodgers (cd.), Workers, Institutions and Economic Growlh in Asia, Ginebra, Organización Internacional del Trabajo (OIT), Instituto Internacional de Estudios Laborales (IIEL)

Buechtemann, C. (1993): Introduction: Employment security and labor markets, C. Buechtemann (ed.) Employment Security and Labor Market Behuvior. Interdisciplinaiy Approaches and International Evidente, Ithaca, ILR Press.

Caire, G. (1994): Labour standards and international trade, W. Sengerberger y D. Campbell (eds.), Internacional Labour Standards and Economic Interdependence, Ginebra, OIT.

Caro Figueroa, A. (1992): Las relaciones de trabajo y la competitividad, Derecho del Trabajo, $\mathrm{N}^{\circ} \mathrm{m}, \mathrm{A}$. Buenos Aires, Editorial La Ley.

Comisión Europea (1994a): Crecimiento, competitividad, empleo. Retos y pistas para entrar en el siglo xxi. Libro blanco, Luxemburgo.

(1994b): La política social europea. Un paso adelante para la Unión, Libro blanco, Luxemburgo, 27 de julio.

De Castro, J.A. (1995): Trade and Labour Standards. Using the Wrong Instruments for the Right Cause. Discussion papers, $\mathrm{N}^{\circ}$ 99, Ginebra, Conferencia de las Naciones Unidas sobre Comercio y Desarrollo (UNCTAD).

Feis, H. (1994): International labour legislaron in the lighl of economic theory, W. Sengerberger y D. Campbell (eds.), International Labour Standards and Economic Interdependence, Ginebra, OIT.

Feldman, S. (1995): Informe sobre las regulaciones y performance económica en Argentina, trabajo presentado a la Reunión sobre regulación del mercado laboral y desempeño económico: Argentina, Brasil y Chile, Santiago de Chile, $0 ; \mathrm{T}$.

FELL (Fundación de Investigaciones Económicas Latinoamericanas) (1992): Costos laborales en el MERCOSUR, Buenos Aires, Ministerio de Relaciones Exteriores y Culto, Programa de las Naciones Unidas para el Desarrollo (PNUD),

Freeman, R. (1994): How labor fares in advanced economies, R. Freeman (ed.), Working Under Different Rules, Nueva York, Russell Sage Foundation.

Gregg, P., S. Machin y A. Manning (1994): High pay, low pay, and labour market efficieney, A. Glyn y D. Miliband (eds.), Paying for Inequality, Londres, Rivcrs Oram Press.
Hikino, T. y A. Amsden (1995): La industrialización tardía en perspectiva histórica, Desarrollo económico, vol. $35, \mathrm{~N}^{\circ} 137$, Buenos Aires, Instituto de Desarrollo Económico y Social (I DES).

Horton, S. R. Kanbur y D. Mazumdar (1994): Labor markets in an era of adjustment An overview, S. Horton, R, Kanbur y D. Mazumdar (eds.), habar Markets in an Era of Adjustment. Case Studies, vol. 2, Washington, D.C., Banco Mundial.

Jacobi, O. (1994): Competítive advantages of Europcan, American and Japanese industrial relations, 4"'Regional Cimgress, Helsinki, International Industrial Relations Association (IIRA).

Kacef, O. y J. Machinea (;995): Cambios recientes en la estructura de precios relativos, Nota, $\mathrm{N}^{\circ} 54$, Buenos Aires, Instituto de Desarrollo Industrial (IDI)

Kosaeoff, B. (1993): La industria argentina: un proceso de reestructuración desarticulada, B. Kosacoff y otros. El desafío de la competitividad. La industria argentina en transformación, Buenos Aires, Comisión Económica para América Latina y el Caribe (CEPAL)/Alianza Editorial.

Marshall, A. (1994): Consecuencias económicas de los regímenes de protección de los trabajadores en América Latina, Revista internacional del trabajo, vol. 113, $\mathrm{N}^{\circ} 1$, Ginebra, OIT, Oficina Internacional del Trabajo.

Maztimdar, D. (1994): The Republic of Korea, S. Horton, R. Kanbur y D. Mazumdar (eds.), Labor Markets in an Era of Adjustment. Case Studies, vol. 2, Washington, D.C., Banco Mundial.

Monza, A. (1993): Costo laboral y competitividad, Esludios del trabajo, $\mathrm{N}^{\circ}$ 6, Buenos Aires, ASET, segundo semestre.

OCDE (Organización de Cooperación y Desarrollo Económicos) (1995): Las normas laborales y la integración económica, Revista de trabajo, $\mathrm{N}^{\circ}$ 5, París.

(1996): Les échanges et les normes du travail, París, marzo.

OIT (Organización Internacional del Trabajo) (1995): El empleo en el mundo, Ginebra.

Park, Y. (1994a): Contemporary challenges to labour standards resulting from globalization: The case of Korea, W Sengerberger y D. Campbell (eds.), International Labour Standards and Economic interdependence, Ginebra, OIT.

(1994b): State regulation, the labour market and economic development: The Republic of Korea, G. Rodgers (eds.), Workers, Institutions and Economic Growth in Asia, Ginebra, OIT, ¡IEL.

Pfaller, A., 1. Gough y G. Therborn (comps.) (1993): Competitividad económica y estado de bienestar: estudio comparativo de cinco países avanzados, Madrid, Ministerio de Trabajo y Asuntos Sociales.

Prezworsky, A. (1987): Marxismo y elección racional, Zona, $\mathrm{N}^{\circ} 45$, Madrid, Editorial Pablo Iglesias.

Schvarzer, J. (1994): La producción argentina en 1994, Buenos Aires, mimeo.

Self, P. (1993): Government by the Marketl, Boulder, Colorado, Westview Press. 
Sengerberger, W. y D, Campbell (eds.) (1994): International Labour Standards and Economic interdependence, Ginebra, OIT.

Standing, G. (1992): Ajuste estructural y políticas laborales: ¿hacia el ajuste social?, Estudios del trabajo, $\mathrm{N}^{\circ} 3$, Ginebra, OIT.

Stiglitz, J. (1987): The causes and consequences of the dependence of quality on price, Journal of Economic Literature, vol. XXV, $\mathrm{N}^{\circ}$ 1, Nashville, Tennessee, American Economic Association. Szretter, H. (1995): Argentina: costo laboral y ventajas competitivas de la industria, Buenos Aires, mimeo.
Tokman, V. y D. Martínez (1995): The impact of labour costs on competitiveness and worker protection in the manufacturing sector of Latin America, trabajo presentado a la Conference on Labour Market Policy in Canada and Latin America Under Economic Integration, Toronto, University of Toronto, diciembre.

Von Potobsky, G. (1995): La regulación laboral a nivel nacional: ¿traba o encuadramiento favorable a un desarrollo sostenido? Relaciones laborales y seguridad social, año I, $N^{\circ} 2$, Buenos Aires, Ediciones Interoceánicas S.A. 